\title{
Hallux valgus-a physiotherapy study with salt solution and ultrasound
}

\begin{abstract}
Introduction: Hallux valgus is a common and disabling condition. This is one of the most common deformations of the human foot. It causes pain, walking difficulties and can be problematic for choosing the proper footwear. Physiotherapy, even when it does not result in correction of deformation, can still reduce pain, improve gait quality and comfort in wearing shoes.
\end{abstract}

Objective: This work means to introduce a new physiotherapy treatment with the use of a salt solution associated ultrasound therapy as a case study.

Methodology: After kinetic-functional evaluation, the patient that had bilateral moderate hallux valgus not received any specific treatment. He was submitted a week session with $20 \%$ copper sulfate solution thought of therapeutic ultrasound and the use of elastic bandage. To have flexion and extension measures were used a goniometer. $\mathrm{X}$-ray in antero-posterior, lateral, and oblique of the foot or the angle to visualize the deformity and the reduction caused.

Results: According to Borg scale for pain in the five sessions no pain was related. It was use elastic therapeutic bandage to sustain the foot under the metatarsus in both members. In the seventh day he wears closed shoes without bother. X-ray shows reduction of the bone protuberance on the Hallux.

Conclusion: Repeated treatment of therapeutic ultrasound with copper sulfate solution, associated with the use of elastic therapeutic bandage may be used as a complementary treatment method for moderate hallux valgus.

Keywords: hallus valgus, physiotherapy, complementary treatment, ultrasound, copper sulfate solution, $\mathrm{x}$-ray, kinetic-functional, teraband
Volume 4 Issue I - 2018

\author{
Anaildes Pamplona,' Sebastião David Santos \\ Filho ${ }^{2}$ \\ IPhysiotherapist, Universidade Potiguar, Brazil \\ 2Physiotherapist, Universidad Mauricio de Nassau, Brazil
}

Correspondence: Sebastião David Santos Filho, Physiotherapist, Universidade Mauricio de Nassau, Natal, RN, Brazil, Email sebastiao.filho@mauriciodenassau.edu.br

Received: February 01, 2018| Published: February I5, 2018

\section{Introduction}

Hallux valgus is a common and disabling condition. ${ }^{1}$ Hallux valgus is one of the most common deformations of the human foot. It causes pain, walking difficulties and can be problematic for choosing the proper footwear. According to the definition it is described as a lateral deviation of the great toe at the metatarsophalangeal joint. Hallux valgus is diagnosed when the hallux valgus angle is greater than 15 degrees.

The aim of surgical correction of hallux valgus is to rebalance the first ray and correct the various features of the deformity. ${ }^{2-5}$ Despite the common occurrence of this condition, treatment remains controversial with more than 150 surgical procedures described. ${ }^{5-7}$ Distal first metatarsal osteotomies traditionally have been indicated for correction of mild-to-moderate deformities with an intermetatarsal angle (IMA) as much as $20^{\circ}$ and for correction of the distal metatarsal articular angle (DMAA). ${ }^{5}$

Physiotherapy, even when it does not result in correction of deformation, can still reduce pain, improve gait quality and comfort in wearing shoes. Therefore, patients with hallux valgus should not be exclusively referred for surgery but should also be considered for of the physiotherapy. ${ }^{8}$ This work means to introduce a new physiotherapy treatment with the use of a salt solution associated ultrasound therapy as a case study.

\section{Methodology}

All this methodology has a protocol patent number 00.000.2.2.17.0167152.7. After kinetic-functional evaluation, the patient, AGMS, a 62-year-old man had bilateral moderate hallux valgus, when he walked with shoes, he complained of pain over the medial eminence of the hallux MTP joint, but he had not received any specific treatment. He was submitted 3 minutes day a one session 2 times per week, with the use of $20 \%$ copper sulfate solution, of blue gelatinous form, applied in the fascia, thought of ultrasound (Ibramed, sonopulse) $3 \mathrm{MHz}$ of frequency, continuous, $0,8 \mathrm{~W} / \mathrm{cm}^{2}$ dose, in circular movements to a complete gel absorption. The excess is removed after the session and the use of elastic bandage (Teraband) for articular stability. The patient was re-evaluated before the procedure session. To have flexion and extension measures were used a goniometer. X-ray in antero-posterior, lateral, and oblique of the foot or the angle to visualize the deformity and the reduction caused. The patient received recommendations about the use of shoes and about the walk. If verify better condition is forwarded to X-ray to verify the real reduction of bone growth.

\section{Results}

At the first day of treatment the patient was oriented to 30minutes sand walk at the beach once per week. According to Borg scale for pain in the second day the patient has grade 8 to the left inferior member (LIM) and 4 to the right inferior member. The Borg number falls to 5 in the third session to LIM. In the fifth session no pain was related by patient. It was use elastic therapeutic bandage (teraband) to sustain the foot under the metatarsus in both members. In the seventh day the patient managed to wear closed shoes without bother. In the re-evaluation with $\mathrm{x}$-ray shows reduction of the bone protuberance on the Hallux (Figure $1 \& 2$ ). 


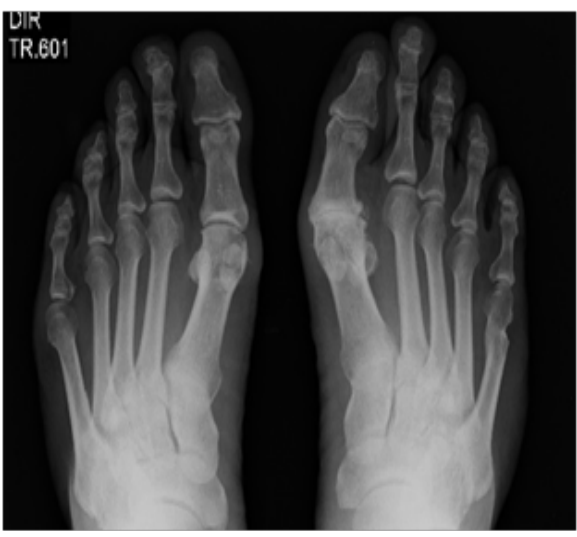

Figure I X-ray of the feet showing the abnormal growing of a protuberance at the hallux in both feet. Note the deformity.

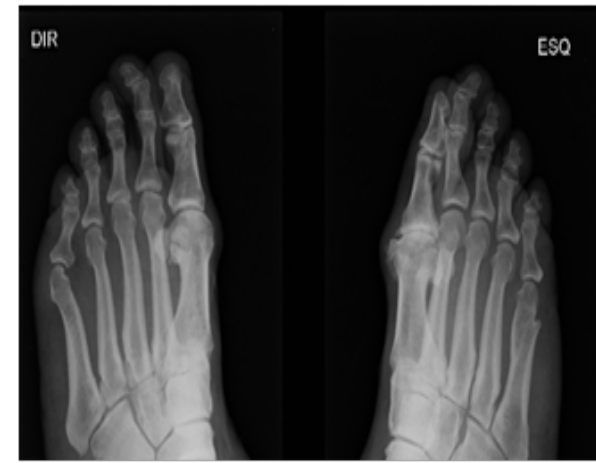

Figure $\mathbf{2}$ X-ray of the feet showing a protuberance at the hallux in both feet that decreased, and the deformity is gone.

\section{Discussion}

Hallux valgus (HV) is defined as an abnormal increase in the lateral deviation angle of the metatarsophalangeal (MTP) joint of the big toe toward the second toe. ${ }^{9}$ The patients refereed pain when using shoes or walking. In the initial assessment of radiographic angles, the $\mathrm{HV}$ angle and the intermetatarsal angle on the right side were $21^{\circ}$ and $15^{\circ}$, respectively. The $\mathrm{HV}$ angle and intermetatarsal angle on the left side were $22^{\circ}$ and $14^{\circ}$, respectively..$^{10}$

The application of elastic therapeutic bandage used in this study is known to have a correction effect on malalignment of the pelvis ${ }^{11}$ and shoulder, ${ }^{12}$ in addition to alleviating pain ${ }^{13,14}$ and grown of lymphatic circulation. ${ }^{15,16}$ Although there were differences with the present study in the method and duration of tape application, we reported that applying kinesiology tape after 23 sessions for this patient with moderate hallux valgus the HV angle effectively reduced as showed in the X-rays images. The walk at the beach sand and the treatment of ultrasound frequency and power with the aid of copper sulfate solution cooperate to the better results.

\section{Conclusion}

Based on the results of this case study, repeated treatment of therapeutic ultrasound with copper sulfate solution, associated with the use of elastic therapeutic bandage may be used as a complementary treatment method for moderate hallux valgus. Further research needs to be conducted to determine whether this treatment will be effective for patients with more severe symptoms, along with comparative studies with other conservative therapies.

\section{Acknowledgements}

We thank the UNP and the UNINASSAU for the support and encouragement of this project.

\section{Conflict of interest}

The authors affirm that no conflict of interest exists about this article.

\section{References}

1. Coughlin MJ, Jones CP. Hallux valgus: demographics, etiology, and radiographic assessment. Foot Ankle Int. 2007;28(7):759-777.

2. Giannini S, Bevoni R, Vannini F, et al. Hallux valgus surgery: the minimally invasive bunion correction. In: Scuderi GR, Tria AJ, editors. Minimally Invasive Surgery in Orthopedics. New York, USA: Springer; 2010. p. 463-471.

3. Giannini S, Ceccarelli F, Bevoni R, et al. Hallux valgus surgery: the minimally invasive bunion correction (SERI). Tech Foot Ankle Surg. $2003 ; 2(1): 11-20$.

4. Giannini S, Faldini C, Vannini F, et al. The minimally invasive osteotomy "S.E.R.I." (simple, effective, rapid, inexpensive) for correction of bunionette deformity. Foot Ankle Int. 2008;29(3):282-286.

5. Giannini S, Vannini F, Faldini $\mathrm{C}$, et al. The minimally invasive hallux valgus correction (S.E.R.I.). Interactive Surgery. 2007;2(1):17-23.

6. Bauer T, de Lavigne C, Biau D, et al. Percutaneous hallux valgus surgery: a prospective multicenter study of 189 cases. Orthop Clin North Am. 2009;40(4):505-514.

7. Oh IS, Kim MK, Lim KY, et al. Modified technique of distal metatarsal osteotomy for hallux valgus. Foot Ankle Int. 2007;28(4):527-528.

8. Saro C, Andren B, Wildemyr Z, et al. Outcome after distal metatarsal osteotomy for hallux valgus: a prospective randomized controlled trial of two methods. Foot Ankle Int. 2007;28(7):778-787.

9. Mortka K, Lisiński P. Hallux valgus-a case for a physiotherapist or only for a surgeon? Literature review. J Phys Ther Sci. 2015;27(10):33033307.

10. Sarwark JF. Essentials of Musculoskeletal Care. 4th ed. American Academy of Orthopaedic Surgeons. Illinois, USA: Springer; 2010.

11. Lee SM, Lee SH. Effects of balance taping using kinesiology tape in a patient with moderate hallux valgus: a case report. Medicine (Baltimore). 2016;95(46): 53357.

12. Lee JH, Yoo WG. Application of posterior pelvic tilt taping for the treatment of chronic low back pain with sacroiliac joint dysfunction and increased sacral horizontal angle. Phys Ther Sport. 2012;13(4):279-285.

13. Gak HB, Lee JH, Kim HD. Efficacy of kinesiology taping for recovery of dominant upper back pain in female sedentary worker having a rounded shoulder posture. Technol Health Care. 2013;21(6):607-612.

14. Lee JH, Yoo WG. Treatment of chronic Achilles tendon pain by Kinesio taping in an amateur badminton player. Phys Ther Sport. 2012;13(2):115-119.

15. Hwang Bo G, Lee JH. Effects of kinesio taping in a physical therapist with acute low back pain due to patient handling: a case report. Int $J$ Occup Med Environ Health. 2011;24(3):320-323.

16. Shim JY, Lee HR, Lee DC. The use of elastic adhesive tape to promote lymphatic flow in the rabbit hind leg. Yonsei Med J. 2003;44(6):10451052. 